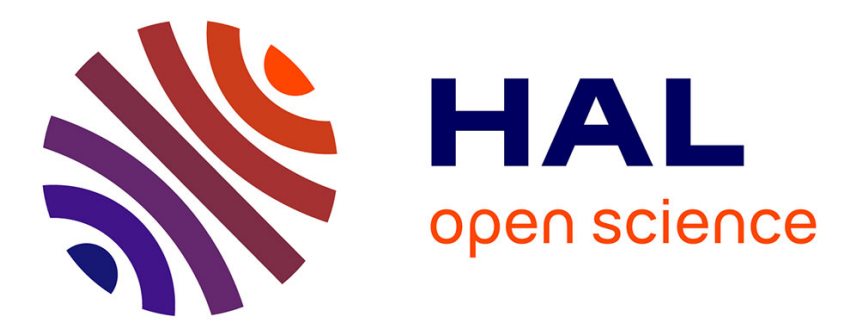

\title{
Evolution de la flore adventice du vignoble de Côte-d'Or sous la pression des techniques d'entretien des cultures
}

\author{
Gilbert Barralis, Gérard Cloquemin, Agnès Guérin
}

\section{To cite this version:}

Gilbert Barralis, Gérard Cloquemin, Agnès Guérin. Evolution de la flore adventice du vignoble de Côte-d'Or sous la pression des techniques d'entretien des cultures. Agronomie, 1983, 3 (6), pp.585-594. hal-00884547

\section{HAL Id: hal-00884547 \\ https://hal.science/hal-00884547}

Submitted on 1 Jan 1983

HAL is a multi-disciplinary open access archive for the deposit and dissemination of scientific research documents, whether they are published or not. The documents may come from teaching and research institutions in France or abroad, or from public or private research centers.
L'archive ouverte pluridisciplinaire HAL, est destinée au dépôt et à la diffusion de documents scientifiques de niveau recherche, publiés ou non, émanant des établissements d'enseignement et de recherche français ou étrangers, des laboratoires publics ou privés. 


\title{
Evolution de la flore adventice du vignoble de Côte-d'Or sous la pression des techniques d'entretien des cultures
}

\author{
Gilbert BARRALIS, Gérard CLOQUEMIN (*) \& Agnès GUERIN (**) \\ I.N.R.A., Laboratoire de Malherbologie, BV 1540, F 21034 Dijon Cedex
}

RÉSUMÉ

\begin{abstract}
Les auteurs présentent la situation actuelle de la flore adventice du vignoble de Côte-d'Or en fonction du mode de désherbage.

La comparaison de cette situation avec celle qui existait 10 ans plus tôt montre que l'importance relative des différentes cspèces a évolué en fonction des techniques d'entretien.

En l'absence de désherbage chimique, comme c'était le cas en 1969 , la flore adventice n'a pratiquement pas varié. En revanche, l'introduction du désherbage chimique depuis cette date a permis l'implantation d'espèces rudérales ou forestières comme Cornus sanguinea, Epilobium tetragonum, Hedera helix, Rubia peregrina... et facilité l'extension de l'ensemble des espèces pérennes comme Allium spp., Muscari spp., Rubus spp. ainsi que de quelques espèces aujourd'hui résistantes aux herbicides comme Senecio vulgaris.
\end{abstract}

Mots clés additionnels : Mauvaises herbes, Vigne, Phytoécologie, Evolution des communautés.

\begin{abstract}
Changes in the weed flora of Côte-d'Or vineyards in relation to weed control methods.
The present situation of the weed flora in Côte-d'Or vineyards is reviewed in relation to weeding methods. Comparison of this situation with that existing 10 years ago shows that the proportion of the different species has been affected by weed control methods.

In the absence of chemical weeding, the weed flora has not changed since 1969. However, the introduction of weed killers after this date has allowed colonization by certain ruderal or forest species like Cornus sanguinea, Epilobium tetragonum, Hedera helix, Rubia peregrina... and has facilitcd the spread of all the perennial species like Allium spp., Muscari spp., Rubus spp., as well as species resistant to herbicides like Senecio vulgaris.
\end{abstract}

Additional key-words : Weeds, Grapevine, Phytoecology, Community change.

\section{INTRODUCTION}

Depuis une trentaine d'années, l'évolution des techniques culturales et, en particulier, la généralisation du désherbage chimique comme moyen de lutte contre les mauvaises herbes a profondément marqué la flore adventice des cultures annuelles assolécs.

Dans le vignoble, de Côte-d'Or en particulier, l'usage des désherbants est plus récent et la région présente ainsi une mosaique de techniques d'entretien des cultures allant des façons aratoires classiques à la non-culture totale.

Nous avons etudié lä flore adventice de ce vignoble et analysć ses variations en fonction des facteurs phytotechniques à partir de relevés floristiques réalisés au cours de la campagne 1981-82.
Ces relevés ont été faits dans les régions de GevreyChambertin-Nuits-St-Georges (63 stations) et MeursaultChassagne-Montrachet ( 39 stations). Ces 2 zones viticoles ont déjà été prospectées en 1968-69 (BARRAlis \& DrouHARD, 1969) ; nous ferons, tout au long de cette étude, référence à ce travail qui nous servira de base de comparaison pour l'étude de l'évolution de la flore adventice sous l'effet des techniques d'entretien des cultures. L'ćtude réalisée en 1968-69 n'avait pas permis di mettre en évidence de différences floristiques entre ces 2 zones proches tant du point de vue géographique que pédo-climatiquc. En 1981 . 82, nous avons aussi réuni cer 2 zones.

\section{METHODOLOGIE}

\section{A. Les relevés floristiques}

Dans le vignoble, la répartition des espèces est généralement hétérogène à cause de la présence d'un rang et d'un inter-rang qui ne sont pas toujours traités de manière
(*) Adresse actuelle : Service de la Protection des Végétaux, Cité administrative, F 67084 Strasbourg Cedex.

(**) Adresse actuelle : Service de la Protection des Végétaux, "La Fenêtre Nord", Route de Vasles - Biard, F 86000 Poitiers. 
identique, et de la présence d'adventices vivaces souvent localisées par taches. De ce fait, la station est définie plutôt par l'homogénéité de ses conditions écologiques que par son homogénéité floristique.

La flore adventice de chaque station a été analysée 4 fois dans l'année, fin novembre, début mars, début mai et fin juin ; à chaque passage, une note globale d'enherbement a été donnée et, à chaque espèce recensée par une prospection systématique de la station, a été attribuée une note d'abondance-dominance selon l'échelle de BRAUN-BLANQUET (tabl. 1) ; cette méthode de notation a été utilisée pour permettre des comparaisons avec les relevés réalisés en 1968-69.

\section{B. Les facteurs stationnels}

Le premier objectif de cette étude étant l'analyse de l'évolution de la flore en fonction des techniques d'entretien des cultures, ce facteur a été minutieusement échantillonné par une enquête auprès des viticulteurs ; 5 classes ont pu être établies :

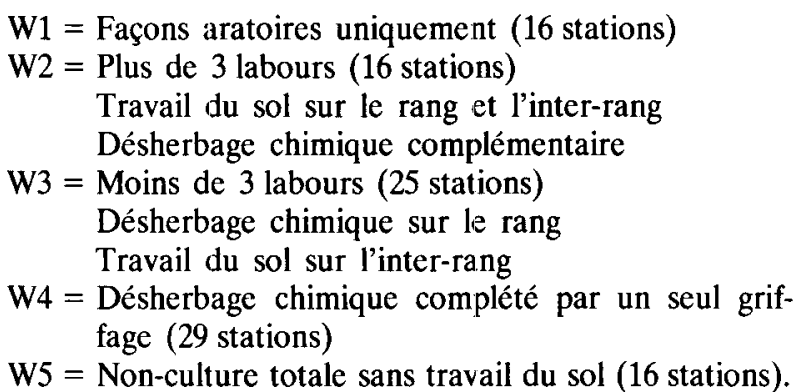

Pour les modalités W4 et W5, le calendrier de traitement est très classique : application de simazine en fin d'hiver et application complémentaire en avril-mai soit d'aminotriazol, soit plus généralement de terbutylazine + terbumeton ou de glyphosate afin de lutter contre les vivaces estivales, liseron et chardon.

\section{Analyse de la végétation}

\section{Analyse globale de la flore}

A chaque classe d'abondance-dominance peut être affecté un coefficient de recouvrement moyen (tabl. 1) qui permet de calculer pour chaque espèce adventice un coefficient de recouvrement (égal à la somme des recouvrements moyens de l'espèce) ainsi qu'un pourcentage de recouvrement (égal au rapport coefficient de recouvrement de l'espèce sur somme des coefficients de recouvrement de toutes les espèces, multiplié par 100) et, pour chaque

\section{TABLEAU 1}

Echelle d'abondance-dominance utilisée. Abundance-dominance scale used.

\begin{tabular}{clc}
\hline Coefficient & Valeur du recouvrement & $\begin{array}{c}\text { Recouvrement } \\
\text { moyen }\end{array}$ \\
\hline+ & Faible & $0,1 \%$ \\
1 & Inférieur à $10 \%$ & 5,0 \\
2 & Compris entre 10 et $25 \%$ & 17,5 \\
3 & Compris entre 25 et $50 \%$ & 37,5 \\
4 & Compris entre 50 et $75 \%$ & 62,5 \\
5 & Compris entre 75 et $100 \%$ & 87,5 \\
\hline
\end{tabular}

station, un recouvrement stationnel moyen (égal à la somme des recouvrements moyens de toutes les espèces divisée par le nombre de stations).

\section{Analyse phytoécologique}

La mise en évidence des corrélations entre la flore et les facteurs d'environnement phytotechniques a été réalisée par la méthode des profils écologiques (GOUNOD, 1969) complétée par l'analyse de l'information mutuelle espècefacteur (GODRON, 1968 ; GUILLERM, 1971) et par l'analyse factorielle des correspondances (BENZEKRI, 1973).

\section{RÉSULTATS}

\section{A. La flore adventice du vignoble}

\section{Physionomie générale}

La flore adventice du vignoble se compose de 124 espèces réparties en 40 familles botaniques dont les mieux représentées sont les composées (16 p. 100 des espèces) et les graminées (13 p. 100) ; l'analyse du spectre biologique brut fait apparaître une nette dominance des annuelles (tabl. 2). Du seul point de vue spécifique, la flore adventice n'a pratiquement pas changé depuis 1968: très généralement les espèces qui n'avaient pas été signalées à cette époque ou qui n'ont pas été retrouvées en 1982 sont des espèces rares dont la fréquence est inférieure à 5 p. 100 ; toutefois quelques espèces, dont la fréquence était comprise entre 10 et 20 p. 100 , n'ont pas été retrouvées : Arenaria serpyllifolia, Medicago lupulina, Fallopia convolvulus, alors que d'autres n'avaient pas été citées: Amaranthus retroflexus, Hedera helix, Rubia peregrina, Sedum spp. ainsi que de nombreuses germinations d'espèces ligneuses.

\section{TABLEAU 2}

Spectre biologique de la flore adventice. Biological spectrum of the weed flora.

\begin{tabular}{lc}
\hline \hline & \\
Espèces annuelles & $54,5 \%$ \\
Espèces bisannuelles & 7,4 \\
Espèces pluriannuelles & 17,4 \\
Espèces vivaces & 14,1 \\
Espèces scmi-ligneuses et ligneuses & 6,6 \\
\hline
\end{tabular}

\section{Physionomie saisonnière}

A l'automne, la flore adventice se compose de 99 espèces dont 47 ont une fréquence supérieure à 5 p. 100 ; à cette époque le vignoble est dominé par 2 espèces très fréquentes et abondantes: Senecio vulgaris et. Stellaria media, suivies de loin par 2 autres espèces très fréquentes mais peu abondantes: Convolvulus arvensis et Lamium purpureum (tabl. 3).

En fin d'hiver, 90 espèces, dont 50 avec une fréquence supćrieure à 5 p. 100 , ont été dénombrées. A Senecio vulgaris et Stellaria media toujours largement dominants, s'ajoutent maintenant Veronica hederifolia et, dans une moindre mesure, Erodium cicutarium (tabl. 4).

Au printemps, la composition spécifique est très comparable, avec 98 espèces rencontrées dont 47 avec une fréquence supéricure à 5 p. 100 , mais la végétation est large- 


\section{TABLEAU 3}

Physionomie de la flore d'automne. Aspects of the autumn weed flora.

\begin{tabular}{|c|c|c|c|}
\hline Classe & & $\begin{array}{l}\text { Fréquence } \\
\text { relative }\end{array}$ & $\begin{array}{l}\text { Pourcentage de } \\
\text { recouvrement }\end{array}$ \\
\hline $\mathrm{V}$ & $\begin{array}{l}\text { Senecio vulgaris } \mathrm{L} \text {. } \\
\text { Stellaria media (L.) Vill. } \\
\text { Convolvulus arvensis L. }\end{array}$ & $\begin{array}{l}98 \\
86 \\
79\end{array}$ & $\begin{array}{r}15,70 \\
28,65 \\
5,00\end{array}$ \\
\hline IV & $\begin{array}{l}\text { Lamium purpureum L. } \\
\text { Erodium cicutarium (L.) L'Hérit. }\end{array}$ & $\begin{array}{l}65 \\
64\end{array}$ & $\begin{array}{l}6,44 \\
3,57\end{array}$ \\
\hline III & $\begin{array}{l}\text { Taraxacum officinale Wiggers } \\
\text { Veronica hederifolia L. } \\
\text { Sonchus asper (L.) Hill } \\
\text { Muscari neglectum Guss. in Ten. } \\
\text { Geranium rotundifolium L. }\end{array}$ & $\begin{array}{l}59 \\
50 \\
48 \\
42 \\
41\end{array}$ & $\begin{array}{l}0,39 \\
3,95 \\
1,65 \\
4,72 \\
2,53\end{array}$ \\
\hline II & $\begin{array}{l}\text { Lactuca serriola } \mathrm{L} . \\
\text { Cirsium arvense }(\mathrm{L} .) \text { Scop. } \\
\text { Sonchus oleraceus } \mathrm{L} . \\
\text { Mercurialis annua } \mathrm{L} . \\
\text { Poa annua } \mathrm{L} . \\
\text { Veronica agrestis } \mathrm{L} . \\
\text { Euphorbia helioscopia } \mathrm{L} . \\
\text { Geranium molle } \mathrm{L} . \\
\text { Calendula arvensis } \mathrm{L} .\end{array}$ & $\begin{array}{l}39 \\
39 \\
30 \\
29 \\
25 \\
23 \\
23 \\
22 \\
21\end{array}$ & $\begin{array}{l}0,26 \\
2,41 \\
1,09 \\
1,52 \\
0,38 \\
1,12 \\
0,84 \\
0,73 \\
2,62\end{array}$ \\
\hline I & 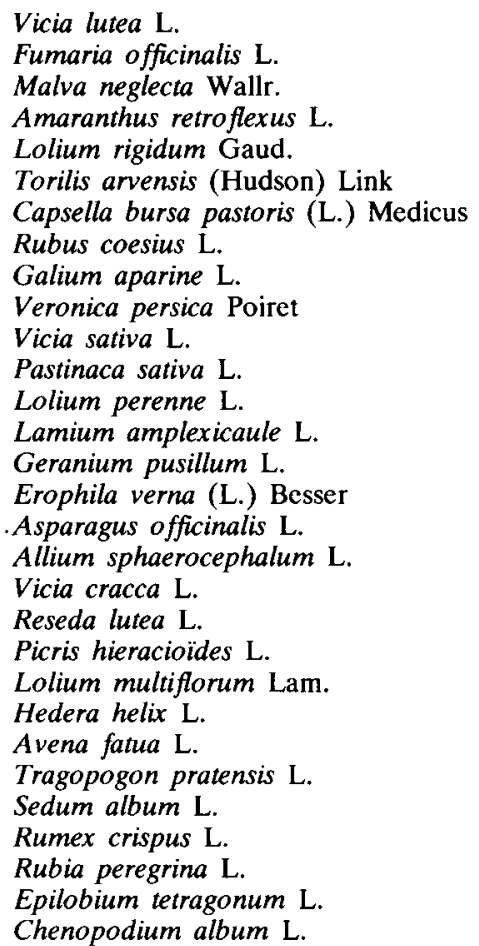 & $\begin{array}{r}20 \\
20 \\
18 \\
18 \\
17 \\
15 \\
15 \\
14 \\
14 \\
13 \\
12 \\
12 \\
11 \\
11 \\
11 \\
11 \\
11 \\
11 \\
10 \\
9 \\
9 \\
8 \\
6 \\
6 \\
5 \\
5 \\
5 \\
5 \\
5 \\
5\end{array}$ & $\begin{array}{l}0,55 \\
0,30 \\
0,62 \\
1,01 \\
0,69 \\
0,48 \\
0,48 \\
0,41 \\
0,02 \\
0,37 \\
1,25 \\
0,33 \\
0,54 \\
0,65 \\
1,14 \\
0,76 \\
0,05 \\
0,86 \\
0,15 \\
0,08 \\
0,08 \\
0,08 \\
0,08 \\
0,08 \\
0,01 \\
0,97 \\
0,01 \\
0,08 \\
0,01 \\
0,01\end{array}$ \\
\hline
\end{tabular}

ment dominée par Convolvulus arvensis, très fréquent et assez abondant, et Senecio vulgaris, moins fréquent mais toujours abondane; on peut y ajouter aussi, moins fréquents et peu abondants: Erodium cicutarium, Muscari neglectum et Stellaria media (tabl. 5).

En été enfin, la flore est un peu moins riche avec 71 espèces dont 32 avec une fréquence supérieure à 5 p. 100 ; Convolvulus arvensis et Senecio vulgaris sont toujours fréquents et abondants, très largement dominants, souvent accompagnés de Cirsium arvense et dans une moindre mesure d'Erodium cicutarium (tabl, 6).

Par rapport à 1968, la physionomie saisonnière du vignoble de Côte-d'Or n'a pas sensiblement changé, les différents faciès se retrouvent avec les mêmes espèces dominantes, toutefois on peut noter la constante régression de Calendula arvensis, espèce caractéristique de l'association des vignes sur sol calcaire (QUANTIN, 1947 ; BRETON, 1956), encore assez fréquente et abondantc en 1968 mais déjà moins qu'en 1945-50. 
TABLEAU 4

Physionomie de la flore de fin d'hiver. Aspects of the late winter flora.

\begin{tabular}{|c|c|c|c|}
\hline Classe & & $\begin{array}{l}\text { Fréquence } \\
\text { relative }\end{array}$ & $\begin{array}{l}\text { Pourcentage de } \\
\text { recouvrement }\end{array}$ \\
\hline V & $\begin{array}{l}\text { Senecio vulgaris L. } \\
\text { Stellaria media (L.) Vill. }\end{array}$ & $\begin{array}{l}89 \\
83\end{array}$ & $\begin{array}{r}7,99 \\
27,62\end{array}$ \\
\hline VI & $\begin{array}{l}\text { Veronica hederifolia } \mathbf{L} . \\
\text { Taraxacum officinale Wiggers }\end{array}$ & $\begin{array}{l}75 \\
75\end{array}$ & $\begin{array}{r}11,21 \\
2,74\end{array}$ \\
\hline III & $\begin{array}{l}\text { Lactuca serriola L. } \\
\text { Erodium cicutarium (L.) L'Hérit. } \\
\text { Sonchus asper (L.) Hill } \\
\text { Veronica agrestis L. } \\
\text { Geranium rotundifolium L. } \\
\text { Muscari neglectum Guss. in Ten. } \\
\text { Lamium purpureum L. } \\
\text { Poa annua L. }\end{array}$ & $\begin{array}{l}56 \\
56 \\
55 \\
54 \\
54 \\
52 \\
51 \\
50\end{array}$ & $\begin{array}{l}2,08 \\
6,13 \\
1,25 \\
2,36 \\
1,21 \\
2,74 \\
6,81 \\
1,63\end{array}$ \\
\hline II & $\begin{array}{l}\text { Lolium perenne } \mathrm{L} . \\
\text { Lamium amplexicaule } \mathrm{L} . \\
\text { Erophila verna (L.) Besser } \\
\text { Vicia lutea } \mathrm{L} . \\
\text { Cirsium arvense (L.) Scop. } \\
\text { Galium aparine } \mathrm{L} . \\
\text { Torilis arvensis (Hudson) Link } \\
\text { Veronica persica Poiret } \\
\text { Bromus hordeaceus } \mathrm{L} . \\
\text { Pastinaca sativa } \mathrm{L} . \\
\text { Crepis setosa Haller } \mathrm{f} .\end{array}$ & $\begin{array}{l}34 \\
34 \\
32 \\
29 \\
28 \\
26 \\
21 \\
21 \\
21 \\
20 \\
20\end{array}$ & $\begin{array}{l}1,94 \\
2,09 \\
3,89 \\
1,94 \\
2,99 \\
1,26 \\
0,23 \\
0,42 \\
0,04 \\
0,66 \\
0,38\end{array}$ \\
\hline I & 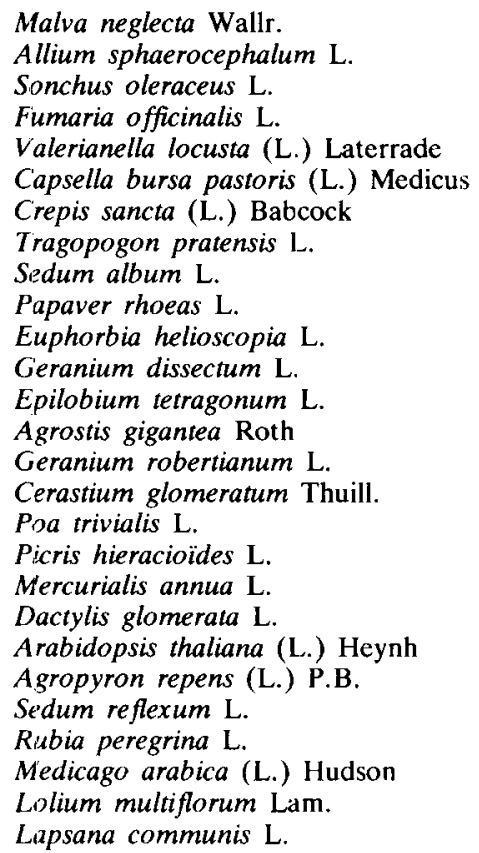 & $\begin{array}{r}18 \\
18 \\
16 \\
16 \\
15 \\
12 \\
11 \\
10 \\
10 \\
10 \\
10 \\
9 \\
9 \\
8 \\
8 \\
8 \\
7 \\
6 \\
6 \\
6 \\
6 \\
6 \\
5 \\
5 \\
5 \\
5\end{array}$ & $\begin{array}{l}0,13 \\
0,13 \\
0,03 \\
0,03 \\
0,95 \\
0,21 \\
0,02 \\
0,02 \\
2,06 \\
0,11 \\
0,02 \\
0,02 \\
0,92 \\
0,11 \\
1,27 \\
0,30 \\
0,01 \\
0,01 \\
0,11 \\
0,01 \\
0,11 \\
0,11 \\
0,74 \\
0,01 \\
0,83 \\
0,01 \\
0,01\end{array}$ \\
\hline
\end{tabular}

\section{B. Influence des techniques d'entretien des cultures}

\section{Sur l'enherbement saisonnier du vignoble}

A l'automne, les parcelles plus ou moins travaillées sont plus sales que l'ensemble des autres parcelles; cette situation est due à un arrêt des travaux du sol dès la fin de l'été afin d'assurer une meilleure portance lors de la récolte, alors que, dans les parcelles en non-culture, les herbicides persistants ont encore un certain effet sur la flore (tabl. $7 a$ ).
En fin d'hiver, la situation est inversée; les parcelles travaillées qui ont été buttées sont dans l'ensemble plus propres que les parcelles en non-culture totale ou partielle, les herbicides ayant perdu à cette époque toute efficacité (tabl. 7b).

A la fin du printemps, dans l'ensemble, les vignes sont propres, mais les parcelles en non-culture présentent un degré de salissement plus élevé que les parcelles travaillées (tabl. 7c). 


\section{TABLEAU 5}

Physionomie de la flore de printemps. Aspects of the spring flora.

\begin{tabular}{|c|c|c|c|}
\hline Classe & & $\begin{array}{l}\text { Fréquence } \\
\text { relative }\end{array}$ & $\begin{array}{l}\text { Pourcentage de } \\
\text { recouvrement }\end{array}$ \\
\hline V & Convolvulus arvensis $\mathrm{L}$. & 82 & 15,05 \\
\hline III & $\begin{array}{l}\text { Muscari neglectum Guss. in Ten. } \\
\text { Stellaria media (L.) Vill. } \\
\text { Senecio vulgaris L. } \\
\text { Taraxacum officinale Wiggers } \\
\text { Erodium cicutarium (L.) L'Hẹrit. }\end{array}$ & $\begin{array}{l}47 \\
46 \\
43 \\
41 \\
40\end{array}$ & $\begin{array}{r}5,73 \\
5,28 \\
16,13 \\
1,74 \\
6,08\end{array}$ \\
\hline II & $\begin{array}{l}\text { Lolium perenne } \mathrm{L} . \\
\text { Geranium rotundifolium } \mathrm{L} . \\
\text { Sonchus asper (L.) Hill } \\
\text { Lamium purpureum L. } \\
\text { Lactuca serriola } \mathrm{L} . \\
\text { Cirsium arvense (L.) Scop. } \\
\text { Poa annua L. } \\
\text { Veronica hederifolia } \mathrm{L} .\end{array}$ & $\begin{array}{l}33 \\
32 \\
29 \\
29 \\
28 \\
28 \\
22 \\
21\end{array}$ & $\begin{array}{l}4,05 \\
2,63 \\
2,31 \\
2,54 \\
1,05 \\
5,66 \\
0,36 \\
0,73\end{array}$ \\
\hline I & 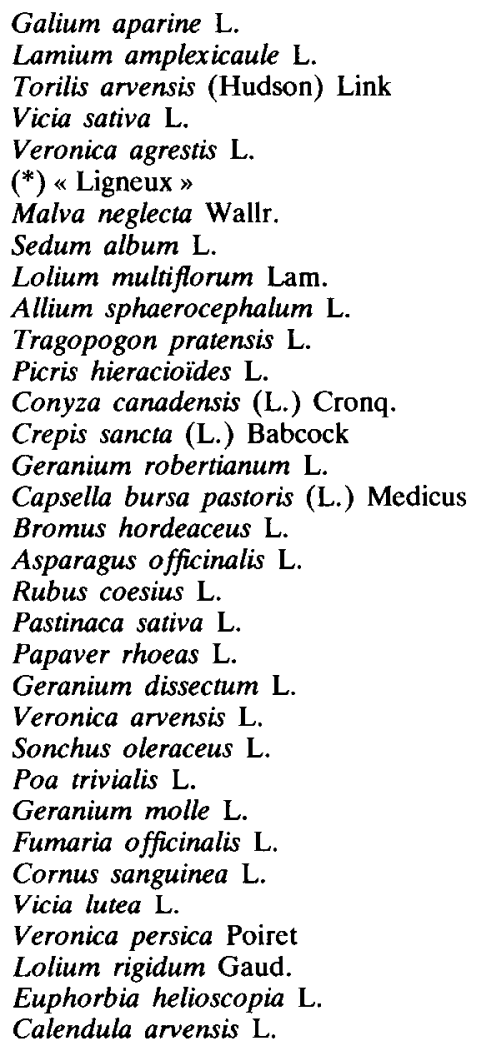 & $\begin{array}{r}19 \\
16 \\
15 \\
14 \\
14 \\
13 \\
13 \\
12 \\
10 \\
10 \\
9 \\
9 \\
9 \\
8 \\
8 \\
8 \\
8 \\
8 \\
7 \\
7 \\
7 \\
7 \\
6 \\
6 \\
6 \\
6 \\
6 \\
6 \\
5 \\
5 \\
5 \\
5 \\
5\end{array}$ & $\begin{array}{l}1,4 \\
0,5 \\
1,01 \\
5 \\
0,19 \\
0,04 \\
1,38 \\
3,25 \\
0,32 \\
0,03 \\
0,17 \\
0,03 \\
1,00 \\
0,02 \\
1,6 \\
0,02 \\
0,02 \\
0,02 \\
0,84 \\
1,96 \\
0,84 \\
0,02 \\
0,17 \\
0,02 \\
0,02 \\
0,84 \\
0,17 \\
0,02 \\
0,69 \\
0,16 \\
0,02 \\
0,02 \\
0,02\end{array}$ \\
\hline
\end{tabular}

$\left({ }^{*}\right)$ Ligneux : Regroupement des germinations d'érables, de pins, de charmes, ...

Enfin, en été, les mauvaises herbes envahissent progressivement le vignoble (tabl. $7 d$ ).

Les valeurs du recouvrement stationnel moyen calculé à partir des coefficients d'abondance-dominance indiquent les mêmes tendances (tabl. 8).

\section{Sur la flore adventice}

Les AFC, réalisées pour chaque époque de relevé sur des matrices espèces-stations et espèces-techniques d'entretien, montrent que l'axe 1, qui absorbe 8 à 10 p. 100 de l'inertie dans le premier type d'analyses et 40 à 50 p. 100 dans le second type, oppose systématiquement les modalités W1 et W5. Les modalités W2, W3 et W4 ont une position intermédiaire, mais $\mathrm{W} 2$ est toujours proche de $\mathrm{W} 1$ et $\mathrm{W} 4$ de $\mathrm{W} 5$, alors que W3 qui recouvre des situations variées a une position médiane généralement plus proche de $\mathrm{W} 2$.

Nous comparerons donc l'effet de la non-culture (W4 + W5) à celui du travail du sol $(\mathrm{W} 1+\mathrm{W} 2)$ sur la flore adventice. 
TABLEAU 6

Physionomie de la flore d'été.

Aspects of the summer flora.

\begin{tabular}{|c|c|c|c|}
\hline Classe & & $\begin{array}{l}\text { Fréquence } \\
\text { relative }\end{array}$ & $\begin{array}{l}\text { Pourcentage de } \\
\text { recouvrement }\end{array}$ \\
\hline $\mathrm{V}$ & $\begin{array}{l}\text { Convolvulus arvensis } \mathrm{L} . \\
\text { Senecio vulgaris } \mathrm{L} .\end{array}$ & $\begin{array}{l}94 \\
84\end{array}$ & $\begin{array}{l}37,94 \\
26,16\end{array}$ \\
\hline II & $\begin{array}{l}\text { Erodium cicutarium (L.) L'Herit. } \\
\text { Taraxacum officinale Wiggers } \\
\text { Cirsium arvense (L.) Scop. } \\
\text { \&igneux }(*) \\
\text { Stellaria media (L.) Vill. } \\
\text { Lolium perenne L. }\end{array}$ & $\begin{array}{l}37 \\
33 \\
33 \\
24 \\
23 \\
20\end{array}$ & $\begin{array}{l}2,96 \\
0,66 \\
8,34 \\
0,17 \\
1,52 \\
1,29\end{array}$ \\
\hline I & 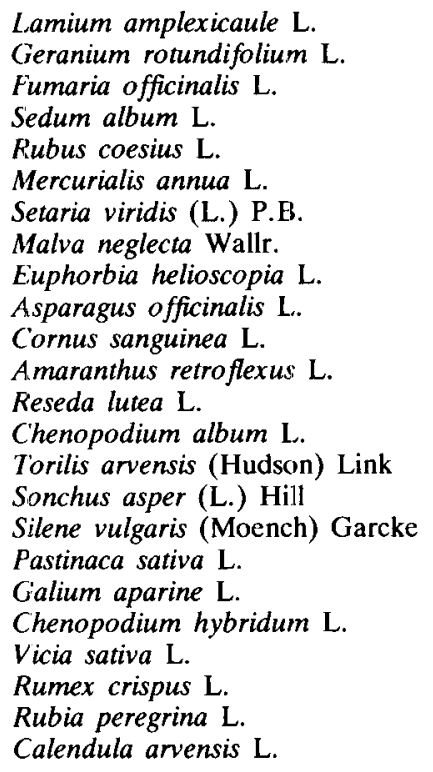 & $\begin{array}{r}14 \\
13 \\
13 \\
11 \\
11 \\
10 \\
9 \\
9 \\
9 \\
9 \\
9 \\
9 \\
7 \\
7 \\
6 \\
6 \\
6 \\
6 \\
6 \\
6 \\
5 \\
5 \\
5 \\
5\end{array}$ & $\begin{array}{l}0,62 \\
0,50 \\
0,73 \\
1,86 \\
1,20 \\
0,02 \\
1,26 \\
0,37 \\
0,25 \\
0,9 \\
0,02 \\
1,44 \\
0,02 \\
0,37 \\
0,01 \\
0,01 \\
0,01 \\
0,54 \\
0,43 \\
0,96 \\
0,13 \\
0,43 \\
0,89 \\
0,36\end{array}$ \\
\hline
\end{tabular}

$\left({ }^{*}\right)$ Regroupement des germinations d'érables, de pins, de charmes...

TABLEAU 7

Fréquence relative des recouvrements par classe de techniques culturales à différentes périodes de l'année. Relative cover in relation to weeding methods at different times of the year.

\begin{tabular}{|c|c|c|c|c|c|c|c|c|c|c|c|c|c|c|c|c|c|c|c|c|}
\hline Epoques & & a) & Autor & nne & & & & Hiv & & & & c) 1 & Printe & mps & & & & 1) Eté & & \\
\hline Recouvrement & $\mathrm{w}_{1}$ & $\mathrm{~W}_{2}$ & $\mathrm{~W}_{3}$ & $\mathrm{~W}_{4}$ & $\mathrm{~W}_{5}$ & $\mathbf{W}_{1}$ & $\mathbf{W}_{2}$ & $\mathrm{~W}_{3}$ & $\mathbf{W}_{4}$ & $\mathrm{~W}_{5}$ & $\mathrm{~W}_{1}$ & $\mathbf{W}_{2}$ & $\mathrm{~W}_{3}$ & $\mathrm{~W}_{4}$ & $\mathbf{W}_{5}$ & $\mathbf{w}_{1}$ & $\mathrm{w}_{2}$ & $\mathrm{w}_{3}$ & $\mathrm{~W}_{4}$ & $\mathrm{~W}_{5}$ \\
\hline $0-1 \%$ & & & 10 & 17 & 23 & 6 & 31 & 4 & 14 & 12 & 71 & 50 & 26 & 31 & 31 & 6 & 25 & 12 & 4 & 6 \\
\hline $1-10 \%$ & & 27 & 45 & 41 & 8 & 41 & 25 & 29 & 31 & 19 & 12 & 25 & 9 & 24 & 19 & 32 & 12 & 32 & 28 & 12 \\
\hline $10-25 \%$ & & 18 & 18 & 26 & 8 & 29 & 31 & 17 & 21 & 12 & 12 & 25 & 22 & 31 & 13 & 37 & 19 & 16 & 50 & 19 \\
\hline $25-50 \%$ & 8 & 22 & 13 & 16 & 46 & 24 & 12 & 12 & 21 & 44 & 5 & & 43 & 14 & 6 & 25 & 25 & 24 & 14 & 44 \\
\hline $50-75 \%$ & & 4 & 8 & & 15 & & & 4 & 10 & & & & & & 31 & & 19 & 12 & 4 & 19 \\
\hline $75-100 \%$ & 92 & 28 & 5 & & & & & 33 & 3 & .12 & & & & & & & & 4 & & \\
\hline
\end{tabular}




\section{TABLEAU 8}

Valeurs du recouvrement stationnel moyen par classe de techniques culturales à différentes périodes de l'année.

Mean cover values of the station in relation to weeding methods at different times of the year.

\begin{tabular}{lrrrr}
\hline \hline \multicolumn{1}{r}{ Epoques } & $\mathbf{W}_{1}$ & $\mathbf{W}_{2}$ & $\mathbf{W}_{4}$ & $\mathbf{W}_{5}$ \\
\hline Automnc & 140 & 93 & 42 & 77 \\
Hiver & 40 & 25 & 44 & 67 \\
Printemps & 10 & 10 & 30 & 47 \\
Eté & 43 & 33 & 33 & 57 \\
\hline
\end{tabular}

a) Espèces dominantes

- Flore d'automne (tabl. 9a).

Par rapport à 1968, on remarque une diminution sensible de la fréquence des véroniques, de Mercurialis annua, Lamium amplexicaule, Fumaria officinalis et une nette augmentation de celle de Lactuca serriola quelles que soient les conditions d'entretien des cultures.

La pratique de la non-culture entraîne une régression de la fréquence de nombreuses mauvaises herbes et en particulier de Cirsium arvense, Lamium amplexicaule et $L$. purpureum, Calendula arvensis, Capsella bursa pastoris, Euphorbia helioscopia et de l'abondance de Stellaria media. En

TABLEAU 9

Fréquence relative des mauvaises herbes dominantes par classe de techniques culturales. Seules sont mentionnées les espèces dont la fréquence dans l'une ou l'autre classe est supérieure à $50 \mathrm{p}$. 100. La valeur indiquée entre parenthèses représente le pourcentage de recouvrement de l'espèce dans la classe lorsqu'il dépasse 10 p. 100.

Relative frequency of the main weeds in relation to weeding methods. Only the species with a frequency higher than $50 \%$ are mentioned. The value in brackets indicates the percentage cover of the species by class when it exceeds $10 \%$.

a) Flore d'automne :

\begin{tabular}{|c|c|c|c|c|c|}
\hline & 1968 & $W_{1}$ & $\mathbf{W}_{2}$ & $\mathrm{~W}_{4}$ & $\mathrm{~W}_{5}$ \\
\hline Senecio vulgaris & $100(7)$ & $100(18)$ & $100(12)$ & $100(16)$ & $92(27)$ \\
\hline Stellaria media & $100(67)$ & $100(51)$ & $95(36)$ & $92(13)$ & $54(2)$ \\
\hline Cirsium arvense & 96 & 100 & 64 & 21 & $<5$ \\
\hline Sonchus annuels & 94 & 83 & 50 & 62 & 46 \\
\hline Convolvulus arvensis & 92 & 83 & 95 & 75 & 62 \\
\hline Veronica agr. + pers. & 89 & 12 & 32 & 25 & 23 \\
\hline Taraxacum officinale & 81 & 75 & 68 & 71 & 54 \\
\hline Veronica hederifolia & 79 & 42 & 55 & 25 & 38 \\
\hline Mercurialis annua & 70 & 50 & 41 & 29 & 23 \\
\hline Lamium purpureum & $69(4)$ & $100(9)$ & $86(11)$ & $50(4)$ & $31(<1)$ \\
\hline Lamium amplexicaule & 68 & 16 & 27 & $<5$ & 0 \\
\hline Calendula arvensis & 65 & 50 & 41 & 0 & $<5$ \\
\hline Geranium pus. + rot. & 65 & 42 & 27 & 59 & 46 \\
\hline Erodium cicutarium & $63(1)$ & $75(1)$ & $45(4)$ & $71(9)$ & $77(5)$ \\
\hline Fumaria officinalis & 62 & 16 & 32 & 16 & $<5$ \\
\hline Lactuca serriola & $<5$ & 83 & 41 & 46 & 31 \\
\hline Capsella bursa pastoris & 25 & 50 & 14 & $<5$ & $<5$ \\
\hline Euphorbia helioscopia & 50 & 33 & 50 & $<5$ & $<5$ \\
\hline Muscari neglectum & 18 & $<5$ & 36 & 42 & 49 \\
\hline Amaranthus spp. & 0 & 0 & $<5$ & 33 & 49 \\
\hline
\end{tabular}

b) Flore d'hiver

\begin{tabular}{lcccc}
\hline Senecio vulgaris & $100(1)$ & $75(3)$ & $90(4)$ & $100(19)$ \\
Stellaria media & $100(40)$ & $94(33)$ & $76(18)$ & $56(3)$ \\
Cirsium arvense & $76(18)$ & $31(5)$ & $<5(<1)$ & $<5(<1)$ \\
Sonchus annuels & 71 & 37 & 59 & 50 \\
Veronica agr. + pers. & 65 & 50 & 41 & 81 \\
Taraxacum officinale & 82 & 63 & 86 & 81 \\
Veronica hederifolia & $71(11)$ & $88(17)$ & $76(18)$ & $63(5)$ \\
Lamium purpureum & 41 & 44 & 52 & 44 \\
Lamium amplexicaule & 53 & 38 & 21 & 37 \\
Geranium pus. + rot. & 47 & 19 & 76 & 52 \\
Erodium cicutarium & $29(<1)$ & $56(9)$ & $69(7)$ & $75(14)$ \\
Lactuca serriola & 53 & 31 & 62 & 50 \\
Muscari neglectum & 29 & 50 & 59 & 81 \\
Poa annua & 35 & 50 & 52 & 37 \\
Erophila verna & 0 & $<5$ & 62 & 59 \\
Vicia spp. & $<5$ & $<5$ & 27 & 56 \\
Lolium perenne & 19 & 12 & 38 & 56 \\
Galium aparine & $<5$ & 12 & & \\
\hline
\end{tabular}


TABLEAU 9 (suite)

c) Flore de printemps

\begin{tabular}{|c|c|c|c|c|c|}
\hline & 1968 & $\mathrm{~W}_{1}$ & $\mathbf{W}_{2}$ & $W_{4}$ & $\mathbf{W}_{5}$ \\
\hline Stellaria media & $100(47)$ & $60(9)$ & $31(<1)$ & $41(4)$ & 0 \\
\hline Convolvulus arvensis & $100(5)$ & $73(24)$ & $81(70)$ & $79(13)$ & $94(8)$ \\
\hline Cirsium arvense & 97 (11) & $60(30)$ & $31(9)$ & $<5(1)$ & $<5(<1)$ \\
\hline Senecio vulgaris & $97(1)$ & $33(6)$ & $12(3)$ & $55(24)$ & $44(25)$ \\
\hline Veronica hederifolia & $94(17)$ & $20(<1)$ & $31(<1)$ & $21(<1)$ & $<5(<1)$ \\
\hline Taraxacum officinale & 94 & 33 & 12 & 55 & 25 \\
\hline Veronica agr. + pers. & 88 & 13 & $<5$ & $<5$ & $<5$ \\
\hline Lamium purpureum & 83 & 33 & 19 & 31 & $<5$ \\
\hline Bromus sterilis & 74 & 0 & 0 & $<5$ & 12 \\
\hline Crepis sancta & 74 & 0 & 0 & $<5$ & 0 \\
\hline Erodium cicutarium & 72 & 20 & 25 & 45 & 44 \\
\hline Poa annua & 71 & 0 & $<5$ & 21 & 12 \\
\hline Geranium pus. $\dagger^{-}$rot. & 68 & $<5$ & 12 & 48 & 25 \\
\hline Sonchus annuels & 68 & 40 & $<5$ & 31 & 19 \\
\hline Muscari neglectum & 55 & 0 & 50 & 55 & 81 \\
\hline Lolium perenne & 23 & $<5$ & $<5$ & 49 & 31 \\
\hline
\end{tabular}

d) Flore d'été

\begin{tabular}{|c|c|c|c|c|c|}
\hline Convolvulus arvensis & $100(50)$ & $100(40)$ & $100(78)$ & $93(30)$ & $88(21)$ \\
\hline Cirsium arvense & $100(21)$ & $81(25)$ & $44(10)$ & $<5(<1)$ & $<5(<1)$ \\
\hline Senecio vulgaris & $94(10)$ & $69(7)$ & $69(7)$ & $93(42)$ & $100(40)$ \\
\hline Stellaria media & 92 & 56 & $<5$ & 18 & 0 \\
\hline Mercurialis annua & 76 & 12 & $<5$ & 7 & 12 \\
\hline Chenopodium album & 66 & 19 & 0 & 0 & 19 \\
\hline Taraxacum officinale & 66 & 31 & 37 & 42 & 19 \\
\hline Calendula arvensis & 63 & $<5$ & $<5$ & 0 & 0 \\
\hline Fumaria officinalis & 54 & 38 & 0 & 7 & $<5$ \\
\hline Jeunes plantes de ligneux & 0 & 25 & 12 & 50 & 19 \\
\hline Erodium cicutarium & 39 & 19 & 25 & 46 & 50 \\
\hline
\end{tabular}

revanche, Amaranthus retroflexus et Muscari neglectum sont nettement favorisées par cette technique.

- Flore de fin d'hiver (tabl. 9b).

A cette époque, les tendances observées à l'automne se confirment, toutefois la fréquence des véroniques, de Taraxacum officinale, de Lamium amplexicaule a augmenté alors que celle de Mercurialis annua, Calendula arvensis, Convolvulus arvensis, Capsella bursa pastoris et Euphorbia helioscopia a fortement diminué. Poa annua est maintenant apparu, sa fréquence se maintient autour de 45 p. 100.

Les différences de fréquences entre parcelles cultivées et parcelles désherbées se retrouvent ; Muscari neglectum ainsi que Galium aparine, Lolium perenne, Vicia spp. sont toujours plus fréquents dans les parcelles en non-culture.

- Flore de fin de printemps (tabl. 9c).

Le nombre d'espèces rencontrées par station est nettement plus faible qu'en 1968 ; cela peut s'expliquer, dans les parcelles en non-culture, par la bonrie efficacité des formulations appliquées au printemps et, dans les parcelles cultivées, par la sécheresse du printemps 1982 et éventuellement par la période qui s'est écoulée entre la date de relevé et la date de la dernière intervention mécanique (variable d'une parcelle à l'autre et d'une période d'observation à l'autre).

Dans les parcelles en non-culture, la fréquence de Stellaria media et de Cirsium arvense est toujours plus faible que dans les parcelles travaillées mais, en revanche, cette technique favorise le développement de Muscari neglectum, Lolium perenne, Poa annua, Geranium rotundifolium.

- Flore de début d'été (tabl. $9 d$ ).

Fin mai, on observe toujours les mêmes tendances entre les différentes techniques d'entretien du vignoble.

\section{b) Espèces liées aux différentes techniques}

Nous venons de voir parmi les espèces dominantes que certaines d'entre elles caractérisent mieux tel ou tel mode d'entretien du vignoble.

L'analyse des profils écologiques corrigés, classés par information mutuelle décroissante, nous a permis de préciser les espèces adventices caractérisant les différentes modalités d'entretien des cultures et l'AFC espèces-techniques d'entretien de confirmer les relations entre la flore adventice et les deux ensembles $\mathrm{W} 1+\mathrm{W} 2$ et $\mathrm{W} 4+\mathrm{W} 5$.

Les analyses phytoécologiques montrent la liaison positive entre travail du sol et Calendula arvensis, Cirsium arvense, Stellaria media, Euphorbia helioscopia, Fumaria officinalis, Lamium amplexicaule et Lamium purpureum; toutefois, pour ces 4 dernières espèces, les différences de fréquence selon le mode d'entretien n'étaient significatives que pour les relevés d'automne et d'été.

De même la liaison positive entre non-culture et $A m a-$ ranthus retroflexus, Galium aparine, Lolium perenne, Muscari neglectum et Vicia spp. est confirmée. 
En outre, un certain nombre d'espèces dont la fréquence varie entre 50 et 5 p. 100 so révèlent caractériser un mode de désherbage et particulièrement la non-culture (tabl. 10).

\section{TABLEAU 10}

Espèces de fréquence comprises entre 50 et 5 p. 100 caractérisant les différentes techniques d'entretien des cultures.

Species with a frequency between 5 and $50 \%$, characteristic of the different weeding methods.

a) Espèces caractérisant le travail du sol $\left(\mathrm{W}_{1}+\mathrm{W}_{2}\right)$

Malva neglecta

Capsella bursa pastoris

b) Espèces caractérisant la non-culture $\left(W_{4}+W_{5}\right)$

Torilis arvensis

Geranium robertianum

Pastinaca sativa

Allium sphaerocephalum

Rubia peregrina

Asparagus officinalis

Hedera helix

Sedum album

Sedum reflexum

Arabidopsis thaliana

Erophila verna

Epilobium tetragonum

Conyza canadensis

Germination d'espèces ligneuses

Cornus sanguinea

Rubus coesitus

\section{DISCUSSION - CONCLUSION}

L'introduction du désherbage chimique dans le vignoble a provoqué un changement sensible de l'importance relative des espèces commensales de cette culture.

Seules les mauvaises herbes de la classe W1 et, dans une moindre mesure, de la classe $\mathrm{W} 2$ présentent en automne une fréquence et une abondance assez comparables à celles de la flore que nous avions observée en 1968 ; au printemps et en été, la fréquence de la plupart des espèces est inférieure, ces différences pouvant être dues aux conditions climatiques de l'année 1982.

Le désherbage chimique a fait régresser un certain nombre d'espèces sensibles à son action, annuelles comme Stellaria media, Lamium purpureum, Euphorbia helioscopia, ou vivaces comme Cirsium arvense et dans une moindre mesure Convolvulus arvensis dont seule l'abondance a diminué.

En revanche, dans les stations désherbées, différentes catégories d'espèces prennent de plus en plus d'importance. Ce sont :

- des espèces annuelles automnales, qui bénéficient peut-être de la réduction de la concurrence comme Erodium cicutarium ou Geranium spp., et printanières à cycle végétatif court qui échappent aux herbicides comme Arabidopsis thaliana ou Erophila verna;

- des espèces annuelles ou pluriannuelles autrefois localisées en bordure de parcelles, mais à dissémination anémophile importante et qui s'installent facilement dans les terrains non travaillés comme Conyza canadensis ou Epilobium tetragonum;

$\rightarrow$ des espèces annuelles présentant une résistance du chloroplaste aux triazines comme Senecio vulgaris.
L'apparition de biotypes résistants à cette famille d'herbicides est assez récente ; en France, ce phénomène particulièrement important dans les cultures de maïs (GASQUEZ et al., 1982) s'étend de plus en plus dans le vignoble. Parmi les espèces signalées comme résistantes dans différentes régions viticoles (Amaranthus retroflexus, Chenopodium album, Conyza canadensis, Epilobium tetragonum, Polygonum persicaria, Senecio vulgaris, Solanum nigrum et Sonchus asper), seules ont été rencontrées à ce jour en Côted'Or, Senecio vulgaris et Conyza camadensis. Le séneçon vulgaire ayant envahi de manière spectaculaire les vignes au cours de la campagne 80/81, nous avons fait des prélèvements de semences dans 30 stations afin d'éprouver la résistance de cette espèce aux triazines. Le tableau 11 montre que, dans différentes stations qui n'ont jamais été traitées chimiquement, les biotypes résistants sont présents ; cette situation est la conséquence de la très large dissémination des semences par le vent. Bien que pour les autres espèces aucun biotýpe résistant n'ait été décelé, il est important de rester vigilant et d'en surveiller l'éventuelle apparition ;

\section{TABLEAU 11}

Répartition des biotypes résistants en fonction des modes d'entretien des parcelles.

Distribution of the resistant biotypes according to weeding method.

\begin{tabular}{lcc}
\hline \multicolumn{1}{c}{$\begin{array}{c}\text { Nature } \\
\text { du mode } \\
\text { d'entretien }\end{array}$} & $\begin{array}{c}\text { Nombre } \\
\text { de stations } \\
\text { échantillonnées }\end{array}$ & $\begin{array}{c}\text { Proportions } \\
\text { de biotypes } \\
\text { résistants }\end{array}$ \\
\hline $\begin{array}{l}\text { Désherbage depuis } \\
\text { plus de } 4 \text { ans }\end{array}$ & 13 & $\begin{array}{c}100 \% \\
\text { environ } 50 \% \\
\text { pas de résistant }\end{array}$ \\
\hline $\begin{array}{l}\text { Aucun traitement } \\
\text { chimique }\end{array}$ & 1 & $\begin{array}{c}\text { environ } 20 \% \\
\text { pas de résistant }\end{array}$ \\
\hline \hline
\end{tabular}

- des espèces vivaces qui sont imparfaitement détruites par les herbicides. Si certaines d'entre elles, comme Allium sphaerocephalum, Muscari neglectum, Rubus coesius, Sedum spp., toujours présentes dans le vignoble, sont aujourd'hui favorisées par l'absence de travail du sol, en revanche plusieurs espèces originaires du milieu environnant (friches, forêts ...) commencent à s'implanter dans le vignoble. C'est le cas d'Asparagus officinale, de Cormus sanguinea, d'Hedera helix, de Rubia peregrina dont les graines sont apportées par les oiseaux et de différentes espèces ligneuses dont les graines sont apportées par le vent (MONTEGUT, 1982). Ce phénomène a été signalé aussi dans le vignoble méditerranéen à la suite de l'extension du désherbage chimique (MAlLLET, 1980).

La non-culture par l'emploi d'herbicides sélectifs présente incontestablement un certain nombre d'avantages techniques ou économiques par rapport au travail mécanique du sol.

Toutefois, comme le montre l'analyse de la flore adventice du vignoble, une évolution de la flore s'est manifestée de 1968 à 1982 sous la pression du désherbage et, plus particulièrement, de la répétition des traitements avec les mêmes matières actives.

L'absence de travail du sol et l'utilisation pendant plusieurs années de formulations insuffisantes sur la plupart des 
espèces vivaces ont favorisé l'installation de certaines espèces.

La fréquence de la plupart d'entre elles est encore faible et il importe, par des traitements localisés spécifiques et efficaces, de limiter l'extension de ces mauvaises herbes avant qu'elles ne deviennent de véritables fléaux.
Le viticulteur qui, pour diverses raisons, opte pour la non-culture doit être attentif aux modifications de la flore qu'induit cette nouvelle technique.

Reçu le 25 novembre 1982. Accepté le 28 février 1983.

\section{RÉFÉRENCES BIBLIOGRAPHIQUES}

Barralls G., Drouhard A., 1969. Quelques aspects de la flore adventice du vignoble de Côte-d'Or. III' Coll. Biol. Mauvaises herbes, 1, 39-61.

Benzekri J.P., 1973. L'analyse des données, Dunod Ed., 615 p. Braun-Blanquet J., 1965. Plant Sociology. Hafner Ed.

Breton R., 1956. Recherches phytosociologiques dans la région de Dijon. Ann. agron., 3, 349-443, et 4, 561-641.

Gasquez J., Barralis G., Aigle N., 198\%. Distribution et extension de la résistance chloroplastique chez les adventices annuelles en France. Agronomie, 2, 119-124.

Godron M., 1968. Quelques applications de la notion de fréquence en écologie végétale. Oecol. Plant., 3, 185-212.

Gounod M., 1969. Méthodes d'étude quantitative de la végétation. Masson Ed.
Guillerm J. L., 1971. Calcul de l'information fournie par un profil écologique et valeur indicatrice des espèces. Oecol. Plant., 6, 209. 225.

Maillet J., 198p. Evolution de la flore adventice des vignobles du Montpellierais sous la pression des herbicides. IV Coll. Biol., Ecol. Syst. Mauvaises Herbes, 359-366.

Montégut J., 1982. Pérennes et vivaces nuisibles en Agriculture. Ed. sous l'égide Monsanto, J. Manuel Ed., Inserenvelop, Aubervilliers, $414 \mathrm{p}$.

Quantin A., 1947. Les associations végétales des cultures sarclées en Bourgogne méridionale. Bull. Soc. Bot. Fr., 94, 320-323.

Tutin T. G. et coll., 1964-1980. Flora Europea. Cambridge University Press. 